\title{
QUASICONFORMAL EXTREMALS OF NON-REGULAR FUNCTIONALS
}

\author{
S.L. Krushkal' \\ Siberian Branch of the Russian Academy of Sciences \\ Institute of Mathematics, 630090 Novosibirsk, Russia \\ Bar-Ilan University, Department of Mathematics and Computer Science \\ 52900 Ramat-Gan, Israel
}

\begin{abstract}
We consider a general extremal problem from the theory of quasiconformal maps with non-regular (and discontinuous) functionals, satisfying a condition of weak subharmonity. The explicit form of extremals of such functionals is established. In this case the extremals do not need to be of Teichmüller type. We establish as an example some important properties of the Grunsky functional.
\end{abstract}

1. Introduction. Let $E$ be a measurable subset of the Riemann sphere $\hat{\mathbf{C}}=\mathbf{C} \cup\{\infty\}$, whose complement $E^{*}=\hat{\mathbf{C}} \backslash E$ has positive measure, and let

$$
B\left(E^{*}\right)=\left\{\mu \in L_{\infty}\left(E^{*}\right):\|\mu\|<1, \mu \mid E=0\right\} .
$$

Denote by $Q(E)$ the class of normalized quasiconformal automorphisms $w^{\mu}(z)$ of the sphere $\hat{\mathbf{C}}$ (with the topology of uniform convergence in spherical metric on $\hat{\mathbf{C}})$ with the Beltrami coefficients $\mu_{w}=\partial_{\bar{z}} w / \partial_{z} w \in B\left(E^{*}\right)$ and arbitrary normalization, which ensures the uniqueness of a solution of the Beltrami equation $\partial_{\bar{z}} w=\mu \partial_{z} w$ if $\mu$ is given.

Let $F\left(w^{\mu}\right)$ be a real functional defined on $Q(E)$, and assume that we have to solve the problem of the maximum of this functional on (compact) subclasses

$$
Q_{k}(E)=\left\{w^{\mu} \in Q(E):\left\|\mu_{w}\right\| \leq k\right\}, \quad 0 \leq k<1 .
$$

The theory of such variational problems for quasiconformal maps is developed only for the continuously differentiable real and holomorphic complex functionals $F$ (see e.g. [1], [2], [4], [8], [11], [13] and the references indicated there). The problems with non-regular functionals have not been studied before.

We shall here investigate the non-regular functionals which are submitted to a certain weakened subharmonity condition only.

1991 Mathematics Subject Classification: Primary 30C62, 30C75, 30C80; Secondary 32F15, $32 \mathrm{G} 15$. 
2. Theorem. Let $F: Q(E) \rightarrow[0, \infty)$ be a bounded functional on $Q(E)$, and let for every fixed $\mu \in B\left(E^{*}\right)$ the function $F\left(w^{t \mu /\|\mu\|_{\infty}}\right)$ be logarithmically subharmonic in $t$ on the unit disc $\Delta=\{t:|t|<1\}$ and the ratio $F\left(w^{t \mu /\|\mu\|}\right) /|t|$ be bounded in a neighbourhood of $t=0$ (by a constant dependent on $\mu$ ). Then, for all $\mu$,

$$
F\left(w^{\mu}\right) \leq A\|\mu\|_{\infty} \quad\left(A=\sup _{Q(E)} F\right),
$$

and if in (1) the equality holds for some $\mu_{0} \neq 0$, then

$$
F\left(w^{t \mu_{0} /\left\|\mu_{0}\right\|}\right)=A|t| \quad \text { for all } t \in \Delta,
$$

or, what is equivalent,

$$
\limsup _{|t| \rightarrow 0} \frac{F\left(w^{t \mu_{0} /\left\|\mu_{0}\right\|}\right)}{|t|}=A .
$$

This theorem essentially sharpens Lehto's majoration principle [10] and a general theorem on the ranges of values for holomorphic functionals proved in $[8$, p. I].

3. Proof of the theorem. We will use the following version of the Schwarz lemma for logarithmically subharmonic functions (cf. [14], [3]).

Lemma. Let a function $u(z): \Delta \rightarrow[0,1)$ be logarithmically subharmonic in the disc $\Delta$ and such that $u(z) /|z|$ is bounded in a neighbourhood of origin. Then

$$
u(z) \leq|z| \quad \text { for all } z \in \Delta
$$

and

$$
\limsup _{|z| \rightarrow 0} \frac{u(z)}{|z|} \leq 1 .
$$

The equality in (4), even for one $z \neq 0$, or in (5) can hold for the function $u(z)=|z|$ only.

Proof. It is well-known that the Green function $g_{\Delta}(z, 0)=\log |z|$ for the disc $\Delta$ with a pole at origin is maximal among all subharmonic functions $v: \Delta \rightarrow$ $[-\infty, 0)$ such that

$$
v(z)=\log |z|+O(1),
$$

where $O(1)$ is bounded in a neighbourhood of $z=0$. (Note that the classical Green function is equal to $-g_{\Delta}(z, 0)$ and it appears as the minimal function for the superharmonic functions $\Delta \rightarrow(0,+\infty])$. 
From hence, taking into account the conditions of the theorem, we immediately obtain

$$
\log u(z) \leq \log |z|
$$

this proves (4).

The investigation of the equality cases requires certain auxiliary considerations. First of all, it follows from the subharmonity of the function $\log u(z)$ in $\Delta$ that the function

$$
\log \frac{u(z)}{|z|}=\log u(z)-\log |z|
$$

is subharmonic in the punctured disc $\Delta \backslash\{0\}$ (since $\log |z|$ is harmonic there). Since the one-point sets are removable for the locally bounded above subharmonic functions, the function

$$
v(z)=\log \frac{u(z)}{|z|}
$$

can be extended to a function subharmonic in the whole disc $\Delta$; we reserve the notation $v$ for the extended function. The function

$$
v_{1}(z)=\frac{u(z)}{|z|}=e^{v(z)}
$$

together with $v(z)$ will then be subharmonc in the whole disc $\Delta$; hence it must be

$$
v_{1}(0) \geq \limsup _{z \rightarrow 0} v_{1}(z) .
$$

Returning to (4), we see that it follows from the availability of the equality $u\left(z_{0}\right)=\left|z_{0}\right|$ at some point $z_{0} \neq 0$ that the function $v_{1}(z)$ attains at this point its maximum equal to 1 , but this is possible only if $v_{1}(z)=1$. If, however, the equality holds for (5), this together with (6) leads to $v_{1}(0)=1$, which again is possible only for $v_{1}(z)=1$. The lemma is proved.

The assertion of the theorem itself follows directly from this lemma applied to the functions

$$
u_{\mu}(t)=F\left(w^{t \mu /\|\mu\|}\right) / A .
$$

Then $\left|u_{\mu}(t)\right| \leq|t|$, and putting $t=\|\mu\|$ we obtain (1) with the equality only for $\mu_{0}$, which satisfy (2). It easily follows from the proof of the lemma that the condition (3) is equivalent to (2) by virtue of the subharmonity of $u_{\mu_{0}}$.

4. Functionals weakly differentiable at the initial point. Now assume that a functional $F$ is differentiable in the sense of Gateau on the set $Q(E)$ at the initial point id: $z \rightarrow z$. This means: for a map

$$
w^{\mu}(z)=z+t H(z)+o(t) \in Q(E), \quad t \in \mathbf{R},
$$


where the variation $H$ is represented by its kernel

$$
h(\mathrm{id}, \zeta)=\frac{1}{\zeta-z}+\text { holomorphic function of } z \text { and } \zeta
$$

according to the formula

$$
H(z)=-\frac{1}{\pi} \iint_{E^{*}} \mu(\zeta) h(\mathrm{id}, \zeta) d \xi d \eta \quad(\zeta=\xi+i \eta)
$$

the equality

$$
F\left(w^{t \mu}\right)=t \operatorname{Re} L(H)+o(t) \quad(F(\mathrm{id})=0)
$$

holds; here $L$ is a continuous complex linear functional on the space of all continuous functions on $\mathbf{C}$. Having $L$ represented as

$$
L(f)=\iint_{\mathbf{C}} f(z) d \lambda(z)
$$

with the corresponding Borel measure $\lambda(z)$, one can extend $L$ to all functions which are integrable on $\mathbf{C}$ with respect to this measure.

We have

$$
\begin{aligned}
\frac{F\left(w^{t \mu_{0}}\right)}{t} & =-\operatorname{Re} L\left(\frac{1}{\pi} \iint_{E^{*}} \mu_{0}(\zeta) h(\mathrm{id}, \zeta) d \xi d h\right)+\frac{o(t)}{t} \\
& =-\operatorname{Re} \frac{1}{\pi} \iint_{E^{*}} \mu_{0}(\zeta) L(h(\mathrm{id}, \zeta)) d \xi d \eta+\frac{o(t)}{t}
\end{aligned}
$$

and it follows from (1), (3) that

$$
\mu_{0}(z)= \begin{cases}\left\|\mu_{0}\right\|_{\infty} \overline{\varphi(z)} /|\varphi(z)|, & z \in E^{*} \\ 0, & z \in E\end{cases}
$$

where

$$
\varphi_{0}(z)=-L(h(\mathrm{id}, z))
$$

and

$$
\frac{1}{\pi} \iint_{E^{*}}|L(h(\mathrm{id}, \zeta))| d x d y=A \quad(z=x+i y) .
$$

Hence, if $F$ is (weakly) differentiable even at one zero point, then all its extremals are maps of Teichmüller type. This means that the Beltrami coefficient $\mu_{0}$ of an extremal map has the form (7): $\left|\mu_{0}(z)\right|=$ const (on $E^{*}$ ) and $\arg \mu_{0}$ is determined by a sufficiently regular function $\varphi_{0}(z)$ (in the problems of the distortion theory $\varphi_{0}$ is usually a rational or holomorphic function).

Note that a similar result in [8] is established under the assumption that the functionals are complex holomorphic. 
5. Non-regular case: the Grunsky functional. Now we show that in the non-regular case the extremals can be of non-Teichmüller type also, even for the functionals which satisfy the condition of the theorem. In other words, the conditions (4), (5) do not imply (7) in this case. We emphasize that we here consider problems of a different type from the Teichmüller problem on the minimization of quasiconformality coefficient.

Let $\Sigma^{\prime}=Q\left(\Delta^{*}\right)$ be the class of univalent functions

$$
w(z)=z+\sum_{n=0}^{\infty} a_{n} z^{-n}
$$

in the region

$$
\Delta^{*}=\{z \in \hat{\mathbf{C}}:|z|>1\}
$$

with a quasiconformal extension to $\bar{\Delta}$. The closure of $\Sigma^{\prime}$ in the topology of uniform convergence on the compacts in $\Delta^{*}$ coincides with the class $\Sigma$ of all the univalent functions in $\Delta^{*}$ with the expansion (8).

It is well-known that a meromorphic function $w$ with the expansion (8) is univalent in $\Delta^{*}$ (i.e. belongs to $\Sigma$ ) if and only if, for its Grunsky coefficients $\alpha_{m n}(w)$ determined from the expansion

$$
\log \frac{w(z)-w(\zeta)}{z-\zeta}=-\sum_{m, n=1}^{\infty} \alpha_{m n}(w) z^{-m} \zeta^{-n}
$$

(where the branch of logarithm is equal to zero for $z=\zeta=\infty$ ), the inequality

$$
\left|\sum_{m, n=1}^{\infty} \sqrt{m n} \alpha_{m n} x_{m} x_{n}\right| \leq\|x\|^{2}
$$

holds for any element $x=\left(x_{n}\right)$ of complex Gilbert space $l^{2}$ with the norm $\|x\|=$ $\left(\sum\left|x_{n}\right|^{2}\right)^{1 / 2}$. The fact that $w$ belongs to $\Sigma^{\prime}$ leads to a sharpened inequality

$$
\left|\sum_{m, n=1}^{\infty} \sqrt{m n} \alpha_{m n}(w) x_{m} x_{n}\right| \leq\left\|\mu_{w}\right\|_{\infty}\|x\|^{2},
$$

but the reverse is not true: from

$$
\left|\sum_{m, n=1}^{\infty} \sqrt{m n} \alpha_{m n}(w) x_{m} x_{n}\right| \leq k\|x\|^{2} \quad \text { for all } x \in l^{2}
$$

it does not at all follow that $w$ would admit quasiconformal extension $\hat{w}$ to $\hat{\mathbf{C}}$ with $\left\|\mu_{\hat{w}}\right\| \leq k$ (see e.g. [9], [5]). 
It is enough in (9), (10) to take $x=\left(x_{n}\right) \in l^{2}$ only with $\|x\|=1$, and this will be assumed hereafter.

Let us consider on $\Sigma^{\prime}$ the functional

$$
F(f)=\sup _{\|x\|=1} \sum_{m, n=1}^{\infty} \sqrt{m n} \alpha_{m n}(f) x_{m} x_{n}
$$

As it was established in [5], [6], the equality

$$
F(f)=\inf \left\{\left\|\mu_{w}\right\|_{\infty}: w^{\mu} \mid \Delta^{*}=f\right\}
$$

(i.e. in (10)) holds for those and only those functions $f \in \Sigma^{\prime}$ which are the restrictions to $\Delta^{*}$ of the quasiconformal automorphisms $w^{\mu_{0}}$ of the sphere $\hat{\mathbf{C}}$ with the Beltrami coefficients $\mu_{0}$ satisfying the condition

$$
\sup _{\varphi \in C^{0}}\left|\iint_{\Delta} \mu_{0}(z) \varphi(z) d x d y\right|=\left\|\mu_{0}\right\|_{\infty}
$$

where $C^{0}$ is the set of holomorphic functions in the disc $\Delta$ with $\iint_{\Delta}|\varphi| d x d y=1$; these are the squares of holomorphic functions: $\varphi=\Phi^{2}$.

\section{The continuity of the Grunsky functional on holomorphic discs} in $B(\Delta)$. Let us show, first of all, that the functional (11) satisfies the conditions of the theorem proved above. This is interesting in itself also, due to the important role of the Grunsky functional in the function theory.

Of these conditions only the following one is not trivial, due to (9) and (10): for every $\mu \in B(\Delta)$ the function

$$
F_{\mu}(t)=F\left(w^{t \mu /\|\mu\|}\right)=\sup _{\|x\|=1}\left|\sum_{m, n=1}^{\infty} \sqrt{m n} \alpha_{m n}\left(w^{t \mu /\|\mu\|}\right) x_{m} x_{n}\right|: \Delta \rightarrow[0,1)
$$

is logarithmically subharmonic in $t$, i.e. the function $\log F_{\mu}(t)$ is upper semicontinuous in $\Delta$ and the inequality for the mean value

$$
\log F_{\mu}\left(t_{0}\right) \leq \frac{1}{2 \pi} \int_{0}^{2 \pi} \log F\left(t_{0}+r e^{t \theta}\right) d \theta, \quad 0 \leq r \leq r_{0}\left(t_{0}\right)
$$

holds for it.

The main difficulty here is to prove the upper semicontinuity. We shall prove that $F_{\mu}(t)$ is in fact continuous in $\Delta$ (and even more); then the subharmonity 
of the function $\log F_{\mu}(t)$ will follow from the fact that this function is the upper envelope for the family of functions $\log \left|h_{x}(t)\right|$ where

$$
h_{x}(t)=\sum_{m, n=1}^{\infty} \sqrt{m n} \alpha_{m n}\left(w^{t \mu /\|\mu\|}\right) x_{m} x_{n}
$$

are holomorphic in $\Delta$ (and $x=\left(x_{n}\right)$ with $\left.\|x\|=1\right)$.

In order to prove the continuity of $F_{\mu}(t)$, we use a method employed in [7], where the functional $F$ was considered on the finite-dimensional Teichmüller spaces.

Fix $\mu \in B(\Delta)$ and denote $\tilde{\mu}=\mu /\|\mu\|_{\infty}$,

$$
C_{t}=w^{t \tilde{\mu}}(|z|=1), \quad \Delta_{t}=w^{t \tilde{\mu}}(\Delta), \quad \Delta_{t}^{*}=w^{t \tilde{\mu}}\left(\Delta^{*}\right) .
$$

Let us associate two functionals with the curves $C_{t}$ :

1) the least positive Fredholm eigenvalue $\lambda_{t}$ defined by the equality

$$
\frac{1}{\lambda_{t}}=\sup \frac{\left|D_{\Delta_{t}}(h)-D_{\Delta_{t}^{*}}(h)\right|}{D_{\Delta_{t}}(h)+D_{\Delta_{t}^{*}}(h)}
$$

where $D_{\Omega}(h)=\iint_{\Omega}\left(\left|h_{x}\right|^{2}+\left|h_{y}\right|^{2}\right) d x d y$ is the Dirichlet integral and the supremum is taken over the set $H_{t}$ of the functions $h$, which are continuous in $\hat{\mathbf{C}}$ and harmonic outside $C_{t}$ with $0<D_{\Delta_{t}}(h)-D_{\Delta_{t}^{*}}(h)<\infty$;

2) Schober's functional

$$
\kappa\left(\Delta_{t}, \Delta_{t}^{*}\right)=\sup _{h \in H_{t}} \frac{D_{\Delta_{t}}(h)}{D_{\Delta_{t}^{*}}(h)} .
$$

On the basis of the known Schiffer-Kühnau result $1 / \lambda_{t}=F_{\mu}(t)$ [12], [10], we have

$$
F_{\mu}(t)=\frac{\max \left\{\kappa\left(\Delta_{t}, \Delta_{t}^{*}\right), \kappa\left(\Delta_{t}^{*}, \Delta_{t}\right)\right\}-1}{\max \left\{\kappa\left(\Delta_{t}, \Delta_{t}^{*}\right), \kappa\left(\Delta_{t}^{*}, \Delta_{t}\right)\right\}+1},
$$

and therefore, for the continuity of $F_{\mu}(t)$, it will be enough to establish the continuous dependence from $t$ (and hence from the curves $C_{t}$ ) of functionals $\kappa\left(\Delta_{t}, \Delta_{t}^{*}\right)$ and $\kappa\left(\Delta_{t}^{*}, \Delta_{t}\right)$.

We shall show that for any sequence $\left\{t_{n}\right\}$ which converges to a point $t_{0} \in \Delta$,

$$
\lim _{n \rightarrow \infty} \kappa\left(\Delta_{t_{n}}, \Delta_{t_{n}}^{*}\right)=\kappa\left(\Delta_{t_{0}}, \Delta_{t_{0}}^{*}\right), \quad \lim _{n \rightarrow \infty} \kappa\left(\Delta_{t_{n}}^{*}, \Delta_{t_{n}}\right)=\kappa\left(\Delta_{t_{0}}^{*}, \Delta_{t_{0}}\right) .
$$

The basic idea of the proof is the same as in [7], although the reasonings are here simplified. Thus we omit some details. 
For the sake of simplicity put the notations

$\Delta_{n}=\Delta_{t_{n}}, \quad \Delta_{n}^{*}=\Delta_{t_{n}}^{*}, \quad C_{n}=C_{t_{n}}, \quad H_{n}=H_{t_{n}}, \quad w_{n}=w^{t_{n} \tilde{\mu}} \quad(n=0,1,2, \ldots)$

and consider the maps

$$
g_{n}^{*}=w_{n} \circ w_{0}^{-1}, \quad n=1,2, \ldots
$$

They are conformal in $\Delta_{0}^{*}$ and have in $\Delta_{0}$ the Beltrami coefficients

$$
\mu_{g_{n}^{*}}(w)=\left(\frac{t_{n}-t_{0}}{1-\bar{t}_{0} t_{n}} \mu \frac{\partial_{z} w_{0}}{\partial_{z} w_{0}}\right) \cdot w_{0}^{-1}
$$

so their quasiconformality coefficients (maximal dilatations) are

$$
K\left(g_{n}^{*}\right)=\frac{1+\left\|\mu_{g_{n}^{*}}\right\|_{\infty}}{1-\left\|\mu_{g_{n}^{*}}\right\|_{\infty}} \rightarrow 1 \quad \text { for } n \rightarrow \infty .
$$

Now fix $h_{n} \in H_{n}$ and define $h_{0}$ as $h_{n} \circ g_{n}^{*} \mid C_{0}$ in $\Delta_{0}$ and as a harmonic extension of the function $h_{n} \circ g_{n}^{*} \mid C_{0}$ in $\Delta_{0}$. Then $h_{0} \in H_{0}$ and, due to the conformality of $g_{n}^{*}$ in $\Delta_{0}^{*}$, we get $D_{\Delta_{0}^{*}}\left(h_{0}\right)=D_{\Delta_{n}^{*}}\left(h_{n}\right)$. On the other hand, the Dirichlet principle and the quasi-invariance of the Dirichlet integral under quasiconformal maps give the relations

$$
D_{\Delta_{0}}\left(h_{0}\right) \leq D_{\Delta_{0}}\left(h_{n} \circ g_{n}^{*}\right) \leq K\left(g_{n}^{*}\right) D_{\Delta_{n}}\left(h_{n}\right) .
$$

Hence we obtain

$$
\kappa\left(\Delta_{0}^{*}, \Delta_{0}\right) \geq \frac{D_{\Delta_{0}^{*}}\left(h_{0}\right)}{D_{\Delta_{0}}\left(h_{0}\right)}=\frac{D_{\Delta_{n}^{*}}\left(h_{n}\right)}{D_{\Delta_{0}}\left(h_{0}\right)} \geq \frac{D_{\Delta_{n}^{*}}\left(h_{n}\right)}{D_{\Delta_{0}}\left(h_{0} \circ g_{n}^{*}\right)} \geq \frac{D_{\Delta_{n}^{*}}\left(h_{n}\right)}{K\left(g_{n}^{*}\right) D_{\Delta_{n}}\left(g_{n}\right)}
$$

and consequently, for all $n=1,2, \ldots$

$$
\kappa\left(\Delta_{0}^{*}, \Delta_{0}\right) \geq \frac{1}{K\left(g_{n}^{*}\right)} \kappa\left(\Delta_{n}^{*}, \Delta_{n}\right) .
$$

Thus, by virtue of (16),

$$
\kappa\left(\Delta_{0}^{*}, \Delta_{0}\right) \geq \limsup _{n \rightarrow \infty} \kappa\left(\Delta_{n}^{*}, \Delta_{n}\right) .
$$

We now establish that

$$
\liminf _{n \rightarrow \infty} \kappa\left(\Delta_{n}^{*}, \Delta_{n}\right) \geq \kappa\left(\Delta_{0}^{*}, \Delta_{0}\right),
$$


and this together with the previous inequality gives the first inequality in (15).

Let $\varepsilon>0$ be arbitrary. We can choose a function $h_{0} \in H_{0}$ such that

$$
\frac{D_{\Delta_{0}^{*}}\left(h_{0}\right)}{D_{\Delta_{0}}\left(h_{0}\right)}>\kappa\left(\Delta_{0}^{*}, \Delta_{0}\right)-\varepsilon
$$

and then transform it into a function $h_{n} \in H_{n}$, setting $h_{n}$ equal to $h_{0} \circ g_{n}^{*-1}$ in $\overline{\Delta_{n}^{*}}$ and in $\Delta_{n}$ equal to a harmonic function with boundary values $h_{0} \circ g_{n}^{*-1}$ on $C_{n}$. Then, using similar considerations, we shall have

$$
\begin{aligned}
\kappa\left(\Delta_{n}^{*}, \Delta_{n}\right) & \geq \frac{D_{\Delta_{n}^{*}}\left(h_{n}\right)}{D_{\Delta_{n}}\left(h_{n}\right)}=\frac{D_{\Delta_{0}^{*}}\left(h_{0}\right)}{D_{\Delta_{n}}\left(h_{n}\right)} \geq \frac{D_{\Delta_{0}^{*}}\left(h_{0}\right)}{K\left(g_{n}^{*}\right) D_{\Delta_{0}}\left(h_{0}\right)} \\
& \geq \frac{1}{K\left(g_{n}^{*}\right)}\left(\kappa\left(\Delta_{0}^{*}, \Delta_{0}\right)-\varepsilon\right) ;
\end{aligned}
$$

hence

$$
\liminf _{n \rightarrow \infty} \kappa\left(\Delta_{n}^{*}, \Delta_{n}\right) \geq \kappa\left(\Delta_{0}^{*}, \Delta_{0}\right)-\varepsilon
$$

and since $\varepsilon$ is arbitrary, we obtain (17).

7. One construction. In order to obtain similar relations for $\kappa\left(\Delta_{n}, \Delta_{n}^{*}\right)$, we must construct the quasiconformal extensions $g_{n}$ of the conformal maps from the region $\Delta_{0}$ onto $\Delta_{n}$ with $K\left(g_{n}\right) \rightarrow 1$ for $n \rightarrow \infty$.

This can be done, for instance, in the following way. Let us extend $\mu$ into $\Delta^{*}$ symmetrically, i.e. take

$$
\mu^{s}(z)= \begin{cases}\frac{\mu(z)}{\mu(1 / z)} z^{2} / \bar{z}^{2} & \text { for } z \in \Delta \\ \text { for } z \in \Delta^{*}\end{cases}
$$

Then $w^{\mu^{s}}$ takes out $\Delta$ and $\Delta^{*}$ into themselves and here $K\left(w^{\mu^{s}}\right)=K\left(w^{\mu}\right)$. Now we define for every $t \in \Delta$ a quasiconformal automorphism

$$
f_{t}(w)=w^{t \tilde{\mu}} \circ\left(w^{t \mu^{s}}\right)^{-1}
$$

of the whole sphere.

Counting its Beltrami coefficient $\mu_{f_{t}}$ according to an elementary formula for the map composition

$$
\mu_{f_{*} \circ f^{-1}} \circ f=\frac{\mu_{f_{*}}-\mu_{f}}{1-\bar{\mu}_{f} \mu_{f_{*}}} \frac{\partial_{z} f}{\overline{\partial_{z} f}}
$$

we see that $f_{t}$ maps conformally the disc $\Delta$ onto the region $\Delta_{t}$ and quasiconformally $\Delta^{*}$ onto $\Delta_{t}^{*}$; in addition,

$$
\mu_{f_{t} \mid \Delta^{*}}(w)=-\left(\mu_{w^{\tilde{t} \mu} \cdot} \frac{\partial_{z} w^{t \tilde{\mu}^{s}}}{\partial_{z} w^{\bar{t} \tilde{\mu}^{s}}}\right) \circ\left(w^{\bar{i} \tilde{\mu}^{s}}\right)^{-1} .
$$


Hence, for any $t^{\prime}, t^{\prime \prime} \in \Delta$, the map

$$
f_{t^{\prime} t^{\prime \prime}} \stackrel{\text { def }}{=} f_{t^{\prime \prime}} \circ f_{t^{\prime}}^{-1}=w^{t^{\prime \prime} \tilde{\mu}} \circ\left(w^{\bar{t}^{\prime \prime} \tilde{\mu}^{s}}\right)^{-1} \circ w^{\bar{t}^{\prime} \tilde{\mu}^{s}} \circ\left(w^{t^{\prime} \tilde{\mu}}\right)^{-1}
$$

has, due to the conformality of $w^{t^{\prime \prime} \tilde{\mu}}$ and $w^{t^{\prime} \tilde{\mu}}$ in $\Delta$, the coefficient of quasiconformality

$$
K\left(f_{t^{\prime} t^{\prime \prime}}\right)=K\left(w^{t^{\prime \prime} \tilde{\mu}_{0}} \circ\left(w^{t^{\prime} \tilde{\mu}}\right)^{-1}\right)=\frac{1+|\tau|}{1-|\tau|}
$$

where

$$
\tau=\left(t^{\prime \prime}-t^{\prime}\right) /\left(1-\vec{t}^{\prime} t^{\prime \prime}\right)
$$

One can see from this that $K\left(f_{t^{\prime} t^{\prime \prime}}\right) \rightarrow 1$ for $t^{\prime \prime} \rightarrow t^{\prime}$.

It remains to put, according to (18),

$$
g_{n}=f_{t_{0} t_{n}} \quad(n=1,2, \ldots)
$$

and then one can apply to $g_{n}$ the considerations similar to those employed above for $g_{n}^{*}$. This gives exactly the second equality from (15).

Remark. The construction from the previous section (and, of course, all reasonings from 6) extends directly to the arbitrary sequences of maps $\left\{w^{\mu_{n}}\right\}$ with $\mu_{n} \in B(D)$, which converge to $\mu_{0} \in B(D)$ in the $L_{\infty}$-norm. Then the function

$$
\log \hat{F}(\mu) \stackrel{\text { def }}{=} \log F\left(w^{\mu}\right): B(\Delta) \rightarrow[-\infty, 0)
$$

is continuous and plurisubharmonic in the ball $B(\Delta)$, and since $F$ depends on the values of the maps $w^{\mu}$ only in the region (disc) of their conformality, it follows that $\hat{F}$ is constant on the fibers of the holomorphic fibering $\Phi: B(\Delta) \rightarrow T(1)$, where $T(1)$ is the universal Teichmüller space. Hence, $\log \hat{F}$ descends as a continuous and plurisubharmonic function to the universal space $T(1)$.

If, however, we consider $\mu$ which are Beltrami differentials with respect to an (arbitrary) Fuchsian group $\Gamma$ in the disc $\Delta$, we analogously find that $\log \hat{F}$ is continuous and plurisubharmonic on the Teichmüller space $T(\Gamma)$ of the group $\Gamma$. (This follows, however, from the above also, because all $T(\Gamma)$ are embedded canonically in $T(1)$.) This supplements, in particular, the results from [6], where only the finite-dimensional Teichmüller spaces were considered.

On the other hand, it follows from what was proved in Section 4 that the functional $F$ (the Grunsky constant) is not differentiable at zero point.

9. An example. Now we shall show that there exist $\mu_{0} \in B(\Delta)$ which satisfy the condition (12) and hence are extremal for the functional (11), with $\left|\mu_{0}(z)\right| \neq$ const in $\Delta$. 
Consider the map

$$
f_{p}(z)=P(x)+i y, \quad z=x+i y
$$

of the half-strip $\Pi_{+}=\{z: 0<x<\infty, 0<y<1\}$ onto itself, which is the streching of this half-strip along the $x$-axis with the variable stretching coefficient

$$
p(x)=\frac{2 x+1}{x+1}
$$

increasing monotonically on $[0, \infty]$ from 1 to 2 . The Beltrami coefficient of this stretching is equal to

$$
\mu_{p}(z)=-\frac{p(x)-1}{p(x)+1}=-\frac{x}{3 x+1} ;
$$

thus $\arg \mu_{p}(z)=\frac{1}{2} \pi$ and $\left\|\mu_{p}\right\|=\frac{1}{3}$.

Let us now take the sequence of the functions

$$
\Phi_{m}(z)=\frac{1}{m} e^{-z / m}, \quad z \in \Pi_{+} \quad(m=1,2, \ldots) .
$$

For it we have

$$
\begin{aligned}
& \iint_{\Pi_{+}}\left|\Phi_{m}(z)\right| d x d y=1 \\
& \begin{aligned}
\left|\iint_{\Pi_{+}} \mu_{p}(z) \Phi_{m}(z) d x d y\right| & =\frac{1}{m}\left|\int_{0}^{1} e^{-i y / m} d y\right| \int_{0}^{\infty} \frac{x e^{-x / m}}{3 x+1} d x \\
& =\frac{1}{m}\left|m\left(1-e^{i / m}\right)\right|\left(\frac{m}{3}-\frac{1}{9} \int_{0}^{\infty} \frac{e^{-x / m}}{x+\frac{1}{3}} d x\right) \\
& =\frac{1}{3}\left(1+O\left(\frac{1}{m}\right)\right)\left(1-\frac{1}{3 m} e^{-1 / 3 m} \int_{3 m}^{\infty} \frac{e^{-t}}{t} d t\right) \\
& =\frac{1}{3}\left(1+O\left(\frac{1}{m}\right)\right)\left(1+O\left(\frac{\log m}{m}\right)\right) .
\end{aligned}
\end{aligned}
$$

With $\Delta$ mapped conformally onto $\Pi_{+}$using a function $z=g(\zeta)$, we construct the map $w^{\mu_{0}} \in \Sigma^{\prime}$ with $\mu_{0}$ equal to zero in $\Delta^{*}$ and equal to $\left(\mu_{p} \circ g\right) \overline{g^{\prime}} / g^{\prime}$ in $\Delta$. Then the corresponding sequence

$$
\varphi_{m}(\zeta)=\left(\Phi_{m} \circ g\right) g^{\prime 2}(\zeta), \quad m=1,2, \ldots,
$$

belongs to the mentioned set $C^{0}$ and is a maximizing sequence for $\left\|m_{0}\right\|$, because it follows from (19) that

$$
\lim _{m \rightarrow \infty}\left|\iint_{\Delta} \mu_{0} \varphi_{m} d \xi d \eta\right|=\frac{1}{3}=\left\|\mu_{0}\right\|_{\infty} .
$$


Hence, the equality (12) is fulfilled and, consequently, $w^{t \mu_{0}}$ is an extremal of the functional (11). Here $\sup _{\Delta}\left|\mu_{0}(z)\right|=\frac{1}{3}$ is attained only at a (unique) boundary point, wherefore $\mu$ can vary in $\Delta$ anyhow (without losing (12)).

Different examples based on the geometrical arguments were constructed by Kühnau (see, e.g. [9]). Note that these examples do not contain the property for dilatation of an extremal map to attain its supremum only at one boundary point. Kühnau informed me that one can obtain such a property also by the corresponding modification of his construction.

The example constructed above is interesting also because it gives an explicitly geodesic holomorphic disc $\left\{\Phi\left(3 t \mu_{0}\right): t \in \Delta\right\}$ in the universal space $T(1)$, which is not a Teichmüller disc and in which the Carathéodory metric coincides with the Teichmüller-Kobayashi metric according to [6].

\section{References}

[1] BelinskiI, P.P.: General properties of quasiconformal mappings. - Nauka, Novosibirsk, 1974 (Russian).

[2] Gutluanskit, V.V., and V.I. RJasanov: On the variational method for quasiconformal mappings. - Siberian Math. J. 28:1, 1987, 81-85.

[3] Klimek, M.: Infinitesimal pseudo-metrics and the Schwarz lemma. - Proc. Amer. Math. Soc. 105, 1989, 134-140.

[4] KRUShKaL', S.L.: Quasiconformal mappings and Riemann surfaces. - Winston, Washington, D.C.; Wiley, New York, 1979.

[5] KrushKal', S.L.: On the Grunsky coefficient conditions. - Siberian Math. J. 28:1, 1987, 138-145.

[6] Krushkal', S.L.: Grunsky coefficient inequalities, Carathéodory metric and extremal quasiconformal mappings. - Comment. Math. Helv. 64, 1989, 650-660.

[7] KrushKaL', S.L.: Strengthening pseudoconvexity of finite-dimensional Teichmüller spaces. - Math. Ann. 290, 1991, 681-687.

[8] Krushkal', S.L., and R. Kühnau: Quasikonforme Abbildungen - neue Methoden und Anwendungen. - Teubner-Texte zur Mathematik 54. Teubner, Leipzig, 1983.

[9] KühnaU, R.: Quasikonforme Fortsetzbarkeit, Fredholmsche Eigenwerte und Grunskysche Koeffizientenbedingungen. - Ann. Acad. Sci. Fenn. Ser. A I Math. 7, 1982, 383-391.

[10] KüHNaU, R.: Wann sind die Grunskysche Koeffizientenbedingungen hinreichend für $Q$ quasikonforme Fortsetzbarkeit? - Comment. Math. Helv. 61, 1986, 290-307.

[11] Leнto, O.: Univalent functions and Teichmüller spaces. - Springer-Verlag, New York, 1986.

[12] Schiffer, M.: Fredholm eigenvalues and Grunsky matrices. - Ann. Polon. Math. 39, 1981, $149-164$.

[13] Sheretov, V.G.: On the theory of extremal quasiconformal mappings. - Math. Sbornik $107: 1,1978,146-151$.

[14] Sibony, N.: A class of hyperbolic manifolds. - In: Recent developments in several complex variables, edited by J.E. Forness. Ann. of Math. Stud. 100, 1981, 357-372. 